\title{
Estimates on front propagation for nonlinear higher-order parabolic equations: An algorithmic approach
}

\author{
JULIAN FISCHER \\ Max Planck Institute for Mathematics in the Sciences, Inselstraße 22, 04103 Leipzig, Germany \\ E-mail: julian.fischer@mis.mpg.de
}

[Received 14 October 2013 and in revised form 28 November 2014]

\begin{abstract}
We present an algorithm for the derivation of lower bounds on support propagation for a certain class of nonlinear parabolic equations. We proceed by combining the ideas in some recent papers by the author with the algorithmic construction of entropies due to Jüngel and Matthes, reducing the problem to a quantifier elimination problem. Due to its complexity, the quantifier elimination problem cannot be solved by present exact algorithms. However, by tackling the quantifier elimination problem numerically, in the case of the thin-film equation we are able to improve recent results by the author in the regime of strong slippage $n \in(1,2)$. For certain second-order doubly nonlinear parabolic equations, we are able to extend the known lower bounds on free boundary propagation to the case of irregular oscillatory initial data. Finally, we apply our method to a sixth-order quantum drift-diffusion equation, resulting in an upper bound on the time which it takes for the support to reach every point in the domain.
\end{abstract}

2010 Mathematics Subject Classification: Primary 35K65; Secondary 35K25, 35B05, 35Q40, 76D08, 35K10.

Keywords: Free boundaries, waiting time phenomenon, degenerate parabolic equation, thin-film equation, doubly nonlinear equation, quantum drift-diffusion equation, computer-assisted proof.

\section{Introduction}

The analysis of support propagation in solutions of nonnegativity-preserving higher-order parabolic equations is a nontrivial issue: In particular, due to the lack of a comparison principle, no lower bounds on support propagation have been available until recently, when the author managed to solve this problem in the case of the thin-film equation [13, 15] and the Derrida-Lebowitz-Speer-Spohn equation [14]. The case of the thin-film equation

$$
\frac{d}{d t} u=-\operatorname{div}\left(u^{n} \nabla \Delta u\right)
$$

(where $n \in \mathbb{R}^{+}$) shows that these difficulties are not just of technical nature, but that the qualitative behaviour of the free boundary depends sensitively on the initial data and the structure of the operator. Let some nonnegative initial data $u_{0} \in H^{1}(\mathbb{R})$ be given and let the leftmost initial free boundary be located at $x_{0}$. Then depending on $n$ and on the growth of the initial data $u_{0}$ near $x_{0}$, the following happens:

- Let $n \in(0,3)$ and (with a grain of salt) $u_{0}(x) \leqslant S \cdot\left(x-x_{0}\right)_{+}^{\frac{4}{n}}$ for some $S>0$. Then a waiting time phenomenon occurs: the left free boundary initially does not advance for some time before it starts moving forward (see [8]). 
- Let $n \in(2,3)$ and $\lim _{\epsilon \rightarrow 0} \epsilon^{-\frac{4}{n}} u_{0}\left(x_{0}+\epsilon\right)=\infty$. Then the left free boundary moves forward immediately (see [12, 15]).

- Let $n \in(1,2)$. Then the initial behaviour of the free boundary does not solely depend on the growth of $u_{0}$ near $x_{0}$ : For example, the stationary solution $u(x, t)=\left(x-x_{0}\right)_{+}^{2}$ grows steeper than $\left(x-x_{0}\right)_{+}^{\frac{4}{n}}$ near $x_{0}$; however, the free boundary is stationary. On the other hand, for any function $g:(0,1) \rightarrow \mathbb{R}$ with $\lim _{h \rightarrow 0} \frac{g(h)}{h^{\frac{4}{n}}}=\infty$, there exists some nonnegative initial data $u_{0}$ with $u_{0}(x) \leqslant g\left(x-x_{0}\right)$ for which the free boundary starts moving forward instantaneously (see [12]).

In the present paper we present an algorithm for the derivation of lower bounds on support propagation for a class of higher-order nonlinear parabolic equations. The algorithm is obtained by combining the ideas developed in the papers [13-15] with the algorithmic construction of entropies due to Jüngel and Matthes [17] (see [6, 18] for further applications of the latter method). We show that one can obtain lower bounds on support propagation by solving a certain quantifier elimination problem; in principle, this quantifier elimination problem could be solved by algorithms which yield an exact solution.

Unfortunately, even in the case of the thin-film equation in one space dimension it turns out that the resulting quantifier elimination problem is intractable with todays exact algorithms and hardware. However, we can use a more direct numerical approach for tackling the quantifier elimination problem; this allows us to improve some of the results in [15]. Namely, for the thinfilm equation in the so-called case of strong slippage $n \in(1,2)$, we obtain improved sufficient conditions for instantaneous forward motion of the free boundary. Additionally, we obtain an idea how the results from $[13,15]$ can be extended to the parameter range $n \in\left[\frac{32}{11}, 3\right)$. Due to technical issues the results in these papers have been limited to $n<\frac{32}{11}$; however, judging by the previous results from the theory of the thin-film equation, one expects the change in qualitative behaviour of solutions to happen at $n=3$. The calculations in [12], which are based on a generalization of the idea obtained in the present paper to multiple spatial dimensions, show that this is indeed the case.

For second-order doubly nonlinear equations of the form

$$
u_{t}=\operatorname{div}\left(u^{m-p+1}|\nabla u|^{p-2} \nabla u\right)
$$

with $p \geqslant 2$ and $m \in(1,2]$, we obtain improved upper bounds on waiting times in case of oscillatory initial data (as compared with the results in [10]).

Finally, as a last application of our method, we analyse the one-dimensional sixth-order quantum drift-diffusion equation considered by Jüngel and Milisic [19] (see the paper by Bukal, Jüngel, and Matthes [5] for the multidimensional version). We show that the support of any solution on $\Omega=(0,1)$ with periodic boundary conditions must touch any point in $\Omega$ within $T=\frac{1}{247000}$. Of course, one might hope to show infinite speed of propagation (the equation being nondegenerate); however, more involved estimates may be necessary for this. See below for a detailed discussion.

\section{Description of the algorithm}

Assume that we are given a parabolic equation of even order $k \geqslant 2$ of the form

$$
\frac{d}{d t} u=u^{\beta+1} P\left(\frac{D u}{u}, \ldots, \frac{D^{k} u}{u}\right),
$$


where $\beta \geqslant 0$ is some real number and where $P$ is a polynomial with the property that in every term on the right-hand side of (2), exactly $k$ derivatives appear (i.e. in the case of one spatial dimension, every monomial $M$ occurring in $P$ must have the form $M(\xi)=\xi^{\eta}$ with $\eta \in\left(\mathbb{N}_{0}\right)^{k}$ and $\sum_{i=1}^{k} i \xi_{i}=$ $k$ ). Note that many nonnegativity-preserving parabolic equations can be written in this form, e.g. the thin-film equation (1) and the Derrida-Lebowitz-Speer-Spohn equation

$$
\frac{d}{d t} u=-\operatorname{div}\left(u \nabla \frac{\Delta \sqrt{u}}{\sqrt{u}}\right) .
$$

Typical phenomena occurring in degenerate parabolic PDEs include finite speed of propagation and waiting time phenomena: if the initial data are compactly supported, the solution will remain compactly supported at any time $t>0$; moreover, if the initial data are "flat enough" at the initial free boundary, the free boundary will not advance for some time before the support of the solution starts spreading. In the present work, we shall derive lower bounds on support propagation and upper bounds on waiting times for nonnegativity-preserving parabolic PDEs of the structure (2).

Denote the initial data for our PDE by $u_{0}$ and the solution by $u$. Let $y \notin \operatorname{supp} u_{0}$ be a point; we define the approaching time $\hat{T}_{y}^{r}$ by

$$
\hat{T}_{y}^{r}:=\inf \left\{T \geqslant 0: \operatorname{dist}\left(y, \bigcup_{t \in[0, T]} \operatorname{supp} u(., t)\right)<r\right\} .
$$

For $x_{0} \in \partial \operatorname{supp} u_{0}$ we define the waiting time $T^{*}$ of $u$ at $x_{0}$ by

$$
T^{*}:=\lim _{\epsilon \rightarrow 0} \inf \left\{t>0: \operatorname{supp} u(., t) \cap B_{\epsilon}\left(x_{0}\right) \not \subset \operatorname{supp} u_{0}\right\} .
$$

The waiting time $T^{*}$ of $u$ at $x_{0}$ therefore is the earliest time at which the support of $u$ starts spreading near $x_{0}$. It is easily seen that the waiting time at $x_{0}$ may be expressed in terms of the approaching times for the points $y \notin \operatorname{supp} u_{0}$ in some neighbourhood of $x_{0}$ : we have

$$
T^{*}=\lim _{\epsilon \rightarrow 0} \inf _{y \in B_{\epsilon}\left(x_{0}\right) \backslash \operatorname{supp} u_{0}} \hat{T}_{y}^{\operatorname{dist}\left(y, \operatorname{supp} u_{0}\right)} .
$$

Therefore upper bounds on waiting times may be deduced by proving upper bounds on approaching times.

The technique of $[13,15]$ for the derivation of upper bounds on waiting times and lower bounds on support propagation for the thin-film equation relies on new monotonicity formulas of the form

$$
\frac{d}{d t} \int u^{1+\alpha}|x-y|^{\gamma} d x \geqslant c \int u^{1+\alpha+n}|x-y|^{\gamma-4} d x,
$$

where $\alpha \in(-1,0)$ and $\gamma<0$. These formulas have been seen to be valid as long as supp $u$ does not touch $y$, i.e. for $t<\sup _{r>0} \hat{T}_{y}^{r}$. Combined with a differential inequality argument due to Chipot and Sideris [7], the monotonicity formulas imply an upper bound on $\hat{T}_{y}^{r}$. In the present work, we use the algorithm of Jüngel and Matthes [17] to prove analogous estimates of the form

$$
\frac{d}{d t} \int u^{1+\alpha}|x-y|^{\gamma} d x \geqslant c \int u^{1+\alpha+\beta}|x-y|^{\gamma-k} d x
$$


for solutions of equations of the structure (2) in the case of one spatial dimension (in theory, the algorithm would also apply to higher space dimensions; however, in practice even for $d=1$ the resulting problem can only be solved by a numerical approach). These analogous estimates then again imply upper bounds on approaching times and, therefore, upper bounds on waiting times.

We now describe our algorithm in detail. It is best explained by the example of the onedimensional porous medium equation

$$
\frac{d}{d t} u=\left(u^{m}\right)_{x x}
$$

$m>1$ (although several steps of the algorithm almost degenerate in case of second-order equations). The one-dimensional porous medium equation can be rewritten as

$$
\frac{d}{d t} u=u^{m}\left(m \frac{u_{x x}}{u}+m(m-1) \frac{u_{x}}{u} \cdot \frac{u_{x}}{u}\right)
$$

which corresponds to the polynomial $P\left(\xi_{1}, \xi_{2}\right):=m \xi_{2}+m(m-1) \xi_{1}^{2}$ and the choice $\beta=m-1$ in formula (2).

Let $d=1$ and $\Omega=\mathbb{R}^{d}=\mathbb{R}$. In short, our algorithm now proceeds as follows:

Step 1: Fix a point $y \in \Omega \backslash \operatorname{supp} u_{0}$, set $\psi:=|x-y|^{\gamma}$ for some $\gamma<0$ which is to be determined, and choose some $r>0$.

Step 2: Formally test the equation (2) with $u^{\alpha}|x-y|^{\gamma}$, where $\alpha \in(-1,0]$ and $\gamma<0$, to obtain an inequality reading

$$
\frac{d}{d t} \int \frac{1}{1+\alpha} u^{1+\alpha}|x-y|^{\gamma} d x \geqslant \int u^{\beta+1+\alpha} P\left(\frac{D u}{u}, \ldots, \frac{D^{k} u}{u}\right)|x-y|^{\gamma} d x
$$

which holds for $t<\hat{T}_{y}^{r}$.

Step 3: Introduce the notation $\xi_{i}$ for $\frac{D^{i} u}{u}$ for each $i \in\{1, \ldots, k\}$. Furthermore introduce the notation $\xi_{0}$ for $(x-y)^{-1}$. Thus the right-hand side of (6) may be replaced by $\int P\left(\xi_{1}, \ldots, \xi_{k}\right) u^{\beta+1+\alpha}|x-y|^{\gamma} d x$.

Step 4: Consider all monomials of the form $M(\xi)=\xi^{\eta}$, with $\eta \in \mathbb{N}_{0}^{k+1}$ satisfying $\eta_{0}+$ $\sum_{i=1}^{k} i \eta_{i}=k$. Then write down all formulas obtained by integration by parts in the integrals

$$
\int M\left((x-y)^{-1}, \frac{D u}{u}, \ldots, \frac{D^{k} u}{u}\right) u^{\beta+1+\alpha}|x-y|^{\gamma} d x,
$$

with a single exception: in case $\eta_{0}=0$, one must not consider integrations by parts which would cause the antiderivative of $|x-y|^{\gamma}$ to appear.

Step 5: Transform the formulas derived in the previous step into the corresponding equations for symbols, i.e. replace $\frac{D^{i} u}{u}$ by $\xi_{i}$; use $\xi_{0}=(x-y)^{-1}$ to rewrite terms of the form $\mid x-$ $\left.y\right|^{\gamma-m}[\operatorname{sign}(x-y)]^{m}$ as $\xi_{0}^{m}|x-y|^{\gamma}$.

Step 6: Check using quantifier elimination whether it is possible to prove that the expression on the right-hand side of (6) is bounded from below by $v \int u^{\beta+1+\alpha}|x-y|^{\gamma-k} d x$ (i.e. $v \int u^{\beta+1+\alpha}|x-y|^{\gamma} \xi_{0}^{k} d x$ ) for some $v>0$ by adding a fixed linear combination of the equations obtained in the previous step. 
Step 7: In case of success, use the resulting differential inequality

$$
\begin{aligned}
\frac{d}{d t} \int \frac{1}{1+\alpha} & u^{1+\alpha}|x-y|^{\gamma} d x \\
& \geqslant v \int u^{\beta+1+\alpha}|x-y|^{\gamma-k} d x \\
& \geqslant v\left(\int u^{1+\alpha}|x-y|^{\gamma} d x\right)^{\frac{\beta+1+\alpha}{1+\alpha}}\left(\int_{\operatorname{supp} u(., t)}|x-y|^{\gamma+k \frac{1+\alpha}{\beta}} d x\right)^{-\frac{\beta}{1+\alpha}}
\end{aligned}
$$

(where the second estimate is a consequence of Hölder's inequality) to obtain blowup of the quantity $\int u^{1+\alpha}|x-y|^{\gamma} d x$ as soon as

$$
\int_{0}^{T} \frac{v \beta}{1+\alpha}\left(\int_{\operatorname{supp} u(., t)}|x-y|^{\gamma+k \frac{1+\alpha}{\beta}} d x\right)^{-\frac{\beta}{1+\alpha}} d t=\left(\int u_{0}^{1+\alpha}|x-y|^{\gamma} d x\right)^{-\frac{\beta}{1+\alpha}}
$$

is satisfied. Note that blowup of the quantity $\int u^{1+\alpha}|x-y|^{\gamma} d x$ at time $T$ implies $\hat{T}_{y}^{r} \leqslant T$. In case $\gamma+k \frac{1+\alpha}{\beta}+d<0$ (we here also consider the case $d>1$ since we shall need that result below), we therefore infer the upper bound

$$
\hat{T}_{y}^{r} \leqslant C(d, \alpha, \beta) \frac{1+\alpha}{\nu \beta}\left(r^{-\gamma-k \frac{1+\alpha}{\beta}-d} \int u_{0}^{1+\alpha}|x-y|^{\gamma} d x\right)^{-\frac{\beta}{1+\alpha}} .
$$

We shall now illustrate the algorithm by the example of the one-dimensional porous medium equation. In this case, Step 3 yields the equation (recall (5))

$$
\frac{d}{d t} \int \frac{1}{1+\alpha} u^{1+\alpha}|x-y|^{\gamma} d x=\int\left(m \xi_{2}+m(m-1) \xi_{1}^{2}\right) u^{m+\alpha}|x-y|^{\gamma} d x .
$$

During Step 4, we obtain the following rules for integration by parts:

$$
\begin{aligned}
& \int u^{m+\alpha} \frac{u_{x x}}{u}|x-y|^{\gamma} d x \\
& \quad=-(\alpha+m-1) \int u^{m+\alpha} \frac{u_{x}}{u} \cdot \frac{u_{x}}{u}|x-y|^{\gamma} d x-\gamma \int u^{m+\alpha} \frac{u_{x}}{u}|x-y|^{\gamma}(x-y)^{-1} d x
\end{aligned}
$$

and

$$
\int u^{m+\alpha} \frac{u_{x}}{u}|x-y|^{\gamma}(x-y)^{-1} d x=-\frac{\gamma-1}{m+\alpha} \int u^{m+\alpha}|x-y|^{\gamma-2} d x .
$$

Rewriting these rules according to Step 5, we obtain

$$
\int\left(\xi_{2}+(m+\alpha-1) \xi_{1}^{2}+\gamma \xi_{0} \xi_{1}\right) u^{m+\alpha}|x-y|^{\gamma} d x=0
$$

and

$$
\int\left(\xi_{0} \xi_{1}+\frac{\gamma-1}{m+\alpha} \xi_{0}^{2}\right) u^{m+\alpha}|x-y|^{\gamma} d x=0
$$


Now we are prepared for formulating the corresponding quantifier elimination problem (Step 6). By adding a general linear combination of the formulas (9) and (10) to the formula (8), we get

$$
\begin{aligned}
\frac{d}{d t} \int \frac{1}{1+\alpha} u^{1+\alpha}|x-y|^{\gamma} d x & \\
=\int\left(m \xi_{2}+m(m-1) \xi_{1}^{2}+\right. & \lambda_{1}\left(\xi_{2}+(m+\alpha-1) \xi_{1}^{2}+\gamma \xi_{0} \xi_{1}\right) \\
& \left.+\lambda_{2}\left(\xi_{0} \xi_{1}+\frac{\gamma-1}{m+\alpha} \xi_{0}^{2}\right)\right) \cdot u^{m+\alpha}|x-y|^{\gamma} d x .
\end{aligned}
$$

Thus it suffices to find $\lambda_{1}, \lambda_{2} \in \mathbb{R}, \alpha \in(-1,0], v>0$, and $\gamma<0$ such that

$$
m \xi_{2}+m(m-1) \xi_{1}^{2}+\lambda_{1}\left(\xi_{2}+(m+\alpha-1) \xi_{1}^{2}+\gamma \xi_{0} \xi_{1}\right)+\lambda_{2}\left(\xi_{0} \xi_{1}+\frac{\gamma-1}{m+\alpha} \xi_{0}^{2}\right) \geqslant v \xi_{0}^{2}
$$

holds for all $\xi_{0}, \xi_{1}, \xi_{2} \in \mathbb{R}$. This is a quantifier elimination problem, which can be solved using algorithms from algebraic geometry. In this special case, we can directly guess the solution $\lambda_{1}=$ $-m$ (as we have no bound on $\xi_{2}$, the contributions of $\xi_{2}$ must vanish), $\lambda_{2}=\gamma m$, which reduces the left-hand side to

$$
-m \alpha \xi_{1}^{2}+\frac{m \gamma(\gamma-1)}{m+\alpha} \xi_{0}^{2}
$$

Therefore $\gamma<0$ and $\alpha \in(-1,0]$ may be chosen arbitrarily and we may set $v=\gamma(\gamma-1) \frac{m}{m+\alpha}$. Estimate (7) then gives

$$
\hat{T}_{y}^{r} \leqslant C(m, \alpha, \gamma)\left(r^{-\gamma-2 \frac{1+\alpha}{m-1}-1} \int u_{0}^{1+\alpha}|x-y|^{\gamma} d x\right)^{-\frac{m-1}{1+\alpha}} .
$$

\section{Application to doubly nonlinear second-order parabolic PDEs}

We now apply our method to the case of doubly nonlinear parabolic PDEs of second order, obtaining an improved version of the estimates on waiting times by Djie [10] for certain parameter ranges. Namely, our upper bounds on waiting times are also valid for highly oscillatory initial data, since in contrast to [10] we do not invoke the comparison principle to compare the solution to radially symmetric and decreasing solutions.

Consider an equation of the form

$$
\frac{d}{d t} u=\operatorname{div}\left(u^{m-p+1}|\nabla u|^{p-2} \nabla u\right)
$$

on $\Omega:=\mathbb{R}^{d}$ with $p \geqslant 2$ and $m>1$. We restrict our attention to weak solutions for this equation with either the regularity $u \log u \in L^{\infty}\left([0, \infty) ; L^{1}\left(\mathbb{R}^{d}\right)\right)$ and $\nabla u^{m / p} \in L^{p}\left(\mathbb{R}^{d} \times[0, \infty)\right)$ or the regularity $u \in L^{\infty}\left([0, \infty) ; L^{1+q}\left(\mathbb{R}^{d}\right)\right)$ and $\nabla u^{(m+q) / p} \in L^{p}\left(\mathbb{R}^{d} \times[0, \infty)\right)$ for some $q>0$. Existence of weak solutions subject to such a regularity condition has been shown e.g. in [1] for a bounded domain; by finite speed of propagation (see e.g. [9]) and extension by zero, this yields 
solutions to the Cauchy problem on any time interval $[0, T]$. By glueing, we obtain solutions on the time interval $[0, \infty)$.

Note that we cannot apply our exact algorithm to the equation (11) since $p$ is not necessarily an integer (and thus, it may be impossible to rewrite the right-hand side in the form (2)); however, we may proceed in a similar spirit.

Our result reads as follows:

THEOREM 1 Let $u_{0} \in L^{1}\left(\mathbb{R}^{d}\right)$ be nonnegative and compactly supported. Suppose that $p \geqslant 2$ and that $m \in(1,2]$. Let $u$ be a weak solution to (11) and let $x_{0} \in \partial \operatorname{supp} u_{0}$. Assume that there exists an open cone with finite height, vertex $x_{0}$, and opening $\Lambda$ which is contained in $\mathbb{R}^{d} \backslash \operatorname{supp} u_{0}$.

Then there exists $\alpha \in(-1,0]$ such that the waiting time $T^{*}$ of $u$ at $x_{0}$ is bounded by

$$
T^{*} \leqslant C(d, m, p, \Lambda) \liminf _{r \rightarrow 0}\left(f_{B_{r}\left(x_{0}\right)}\left|\frac{u_{0}(x)}{r^{2 /(m-1)}}\right|^{1+\alpha} d x\right)^{-\frac{m-1}{1+\alpha}} .
$$

Note that our theorem also provides upper bounds on waiting times for initial data which behave e.g. like $\sin ^{2}\left(x^{-s}\right) x_{+}^{\frac{2}{m-1}}, s>0$, near the initial free boundary. In contrast, the results of [10] do not apply to such cases, at least for $s>0$ large enough.

Reverse estimates (i.e., lower bounds on waiting times) have been obtained in [16, 20]; these results imply that our bounds are optimal up to a constant factor.

Proof. First we would like to show additional regularity of solutions to (11) in order to justify the subsequent computations. To repeat the standard argument, for $-1<\alpha<0$ one can test the equation with $\max (\delta, \min (u, K))^{\alpha}$ (note that this is an admissible test function) to obtain (using a now-standard argument to rearrange the time derivative; see, e.g., [1])

$$
\left.\int_{\mathbb{R}^{d}} \int_{0}^{u} \max (\delta, \min (s, K))^{\alpha} d s d x\right|_{0} ^{T}=-\alpha \int_{0}^{T} \int_{\mathbb{R}^{d}} \chi_{\{\delta<u<K\}} u^{m-p+\alpha}|\nabla u|^{p-2} \nabla u \cdot \nabla u d x d t .
$$

This gives

$$
\begin{aligned}
\int_{0}^{T} \int_{\mathbb{R}^{d}} & \left|\nabla \max (\delta, \min (u, K))^{(m+\alpha) / p}\right|^{p} d x d t \\
& \leqslant C(\alpha) \int_{\mathbb{R}^{d}} K^{\alpha}(u-K)_{+}+\frac{1}{1+\alpha}\left(\min (u, K)^{1+\alpha}-\delta^{1+\alpha}\right)_{+}+\left.\delta^{\alpha} \min (u, \delta) d x\right|_{0} ^{T} .
\end{aligned}
$$

Making use of the finite speed of propagation property (which implies that the set $\operatorname{supp} u(., T)$ is bounded) and letting $\delta \rightarrow 0$ and $K \rightarrow \infty$, we infer $\nabla u^{(m+\alpha) / p} \in L^{p}\left(\mathbb{R}^{d} \times[0, T]\right)$ for any $T>0$.

Now take some general $\alpha \in(-1,0)$ and let $y \in \mathbb{R}^{d} \backslash \operatorname{supp} u_{0}$. Testing the weak formulation of equation (11) with $u^{\alpha}|x-y|^{\gamma}$ (note that one needs standard approximation arguments here analogous to the ones above), we get

$$
\begin{aligned}
\frac{d}{d t} \int \frac{1}{1+\alpha} u^{1+\alpha}|x-y|^{\gamma} d x=-\alpha & \int u^{m-p+\alpha}|\nabla u|^{p}|x-y|^{\gamma} d x \\
& -\gamma \int u^{m-p+1+\alpha}|\nabla u|^{p-2} \nabla u \cdot(x-y)|x-y|^{\gamma-2} d x .
\end{aligned}
$$


We have the following formula for integration by parts:

$$
(\gamma-p+d) \int u^{m+\alpha}|x-y|^{\gamma-p} d x=-(m+\alpha) \int u^{m+\alpha-1} \nabla u \cdot(x-y)|x-y|^{\gamma-p} d x .
$$

This yields

$$
\begin{aligned}
\frac{d}{d t} \int \frac{1}{1+\alpha} u^{1+\alpha}|x-y|^{\gamma} d x=- & \alpha \int u^{m-p+\alpha}|\nabla u|^{p}|x-y|^{\gamma} d x \\
& -\gamma \int u^{m-p+1+\alpha}|\nabla u|^{p-2} \nabla u \cdot(x-y)|x-y|^{\gamma-2} d x \\
& -\lambda(m+\alpha) \int u^{m+\alpha-1} \nabla u \cdot(x-y)|x-y|^{\gamma-p} d x \\
& -\lambda(\gamma-p+d) \int u^{m+\alpha}|x-y|^{\gamma-p} d x,
\end{aligned}
$$

where $\lambda>0$ is arbitrary. We now choose $\lambda:=|\gamma|^{p-1}$. In this case, for a.e. point the terms in the second and the third integral on the right-hand side of the previous formula have opposite sign (since $m>1$ and $\alpha>-1$ and $\gamma<0$ ). Thus, we deduce

$$
\begin{aligned}
& \frac{d}{d t} \int \frac{1}{1+\alpha} u^{1+\alpha}|x-y|^{\gamma} d x \\
& \begin{aligned}
\geqslant-\alpha \int u^{m-p+\alpha}|\nabla u|^{p}|x-y|^{\gamma} d x+\int \min ( & -\gamma u^{m-p+1+\alpha}|\nabla u|^{p-2} \nabla u \cdot(x-y)|x-y|^{\gamma-2}, \\
& \left.-(m+\alpha)|\gamma|^{p-1} u^{m+\alpha-1} \nabla u \cdot(x-y)|x-y|^{\gamma-p}\right) d x \\
& +\left(|\gamma|^{p}-d|\gamma|^{p-1}\right) \int u^{m+\alpha}|x-y|^{\gamma-p} d x .
\end{aligned}
\end{aligned}
$$

As a consequence, for $|\gamma|$ large enough and $\alpha>-1$ close enough to -1 (recall that $m \in(1,2])$, we infer by Young's inequality that

$$
\frac{d}{d t} \int \frac{1}{1+\alpha} u^{1+\alpha}|x-y|^{\gamma} d x \geqslant c \int u^{m+\alpha}|x-y|^{\gamma-p} d x
$$

is satisfied. By the cone condition, for $r>0$ small enough we may choose $y \in B_{r}\left(x_{0}\right)$ with $\operatorname{dist}\left(y, \operatorname{supp} u_{0}\right) \geqslant c(d, \Lambda) r$. We then get by the considerations preceding $(7)$

$$
\hat{T}_{y}^{\operatorname{dist}\left(y, \operatorname{supp} u_{0}\right)} \leqslant C(d, m, \alpha, p, \Lambda)\left(r^{-\gamma-2 \frac{1+\alpha}{m-1}-d} \int u_{0}^{1+\alpha}|x-y|^{\gamma} d x\right)^{-\frac{m-1}{1+\alpha}} .
$$

This finally yields the bound

$$
T^{*} \leqslant C(d, m, \alpha, p, \Lambda) \liminf _{r \rightarrow 0}\left(f_{B_{r}\left(x_{0}\right)}\left|\frac{u_{0}(x)}{r^{2 /(m-1)}}\right|^{1+\alpha} d x\right)^{-\frac{m-1}{1+\alpha}} .
$$




\section{Application to the thin-film equation}

\subsection{Derivation of the quantifier elimination problem}

We now apply our method to the thin-film equation. We may formally test the weak formulation of the thin-film equation with $u^{\alpha}|x-y|^{\gamma}$ and integrate by parts to directly eliminate the symbols corresponding to the third and fourth spatial derivative. This yields

$$
\begin{aligned}
\frac{d}{d t} \frac{1}{1+\alpha} & \int u^{1+\alpha}|x-y|^{\gamma} d x=\int u^{n} u_{x x x}\left(\alpha u^{\alpha-1} u_{x}|x-y|^{\gamma}+u^{\alpha}\left(|x-y|^{\gamma}\right)_{x}\right) d x \\
= & -\alpha(n+\alpha-1) \int u^{n+\alpha-2} u_{x x}\left|u_{x}\right|^{2}|x-y|^{\gamma} d x-\alpha \int u^{n+\alpha-1}\left|u_{x x}\right|^{2}|x-y|^{\gamma} d x \\
& -\gamma \alpha \int u^{n+\alpha-1} u_{x x} u_{x}|x-y|^{\gamma-1} \operatorname{sign}(x-y) d x \\
& -\gamma(n+\alpha) \int u^{n+\alpha-1} u_{x x} u_{x}|x-y|^{\gamma-1} \operatorname{sign}(x-y) d x \\
& -\gamma(\gamma-1) \int u^{n+\alpha} u_{x x}|x-y|^{\gamma-2} d x
\end{aligned}
$$

Note that this equation can be made rigorous by an approximation argument as in [13, 15] (provided that $t<\hat{T}_{y}^{r}$ for some $r>0$ ). Replacing all derivatives of $u$ by the corresponding symbols $\xi_{1}$ to $\xi_{4}$ (and $\xi_{0}$ ), the right-hand side becomes

$$
\begin{aligned}
& -\alpha(n+\alpha-1) \int u^{n+\alpha+1}|x-y|^{\gamma} \xi_{2} \xi_{1}^{2} d x-\alpha \int u^{n+\alpha+1}|x-y|^{\gamma} \xi_{2}^{2} d x \\
& \quad-\gamma(n+2 \alpha) \int u^{n+\alpha+1}|x-y|^{\gamma} \xi_{2} \xi_{1} \xi_{0} d x-\gamma(\gamma-1) \int u^{n+\alpha+1}|x-y|^{\gamma} \xi_{0}^{2} \xi_{2} d x .
\end{aligned}
$$

We now write down all formulas for integration by parts which involve at most second derivatives of $u$ (note that it follows from considerations in [17] that the formulas involving higher-order derivatives of $u$ are not needed; note also that the approximation arguments in [15] justify the formulas for integration by parts which are to follow, at least for solutions to the thin-film equation and a.e. $t>0$ ): We have

$$
\begin{aligned}
3 \int u^{n+\alpha-2} u_{x x}\left|u_{x}\right|^{2}|x-y|^{\gamma} d x=-( & +\alpha-2) \int u^{n+\alpha-3}\left|u_{x}\right|^{4}|x-y|^{\gamma} d x \\
& -\gamma \int u^{n+\alpha-2}\left|u_{x}\right|^{2} u_{x}|x-y|^{\gamma-1} \operatorname{sign}(x-y) d x
\end{aligned}
$$

and

$$
\begin{aligned}
2 \int u^{n+\alpha-1} u_{x x} u_{x}|x-y|^{\gamma-1} & \operatorname{sign}(x-y) d x \\
= & -(n+\alpha-1) \int u^{n+\alpha-2}\left|u_{x}\right|^{2} u_{x}|x-y|^{\gamma-1} \operatorname{sign}(x-y) d x \\
& -(\gamma-1) \int u^{n+\alpha-1}\left|u_{x}\right|^{2}|x-y|^{\gamma-2} d x
\end{aligned}
$$


as well as

$$
\begin{aligned}
\int u^{n+\alpha} u_{x x}|x-y|^{\gamma-2} d x= & -(n+\alpha) \int u^{n+\alpha-1}\left|u_{x}\right|^{2}|x-y|^{\gamma-2} d x \\
& -(\gamma-2) \int u^{n+\alpha} u_{x}|x-y|^{\gamma-3} \operatorname{sign}(x-y) d x
\end{aligned}
$$

and

$$
(n+\alpha+1) \int u^{n+\alpha} u_{x}|x-y|^{\gamma-3} \operatorname{sign}(x-y) d x=-(\gamma-3) \int u^{n+\alpha+1}|x-y|^{\gamma-4} d x .
$$

Using our symbols $\xi_{0}, \xi_{1}, \xi_{2}$, we see that these formulas correspond to

$$
\begin{gathered}
3 \int u^{n+\alpha+1}|x-y|^{\gamma} \xi_{2} \xi_{1}^{2} d x=-(n+\alpha-2) \int u^{n+\alpha+1}|x-y|^{\gamma} \xi_{1}^{4} d x \\
-\gamma \int u^{n+\alpha+1}|x-y|^{\gamma} \xi_{1}^{3} \xi_{0} d x
\end{gathered}
$$

and

$$
\begin{array}{r}
2 \int u^{n+\alpha+1}|x-y|^{\gamma} \xi_{2} \xi_{1} \xi_{0} d x=-(n+\alpha-1) \int u^{n+\alpha+1}|x-y|^{\gamma} \xi_{1}^{3} \xi_{0} d x \\
-(\gamma-1) \int u^{n+\alpha+1}|x-y|^{\gamma} \xi_{1}^{2} \xi_{0}^{2} d x
\end{array}
$$

as well as

$$
\begin{array}{r}
\int u^{n+\alpha+1}|x-y|^{\gamma} \xi_{2} \xi_{0}^{2} d x=-(n+\alpha) \int u^{n+\alpha+1}|x-y|^{\gamma} \xi_{1}^{2} \xi_{0}^{2} d x \\
-(\gamma-2) \int u^{n+\alpha+1}|x-y|^{\gamma} \xi_{1} \xi_{0}^{3} d x
\end{array}
$$

and

$$
(n+\alpha+1) \int u^{n+\alpha+1}|x-y|^{\gamma} \xi_{1} \xi_{0}^{3} d x=-(\gamma-3) \int u^{n+\alpha+1}|x-y|^{\gamma} \xi_{0}^{4} d x .
$$

Our quantifier elimination problem therefore reads: Given some $n>0$, find constants $\alpha, \gamma, \lambda_{1}, \ldots, \lambda_{4}$ such that for any $\xi_{0}, \xi_{1}, \xi_{2} \in \mathbb{R}$ the estimate

$$
\begin{aligned}
& -\alpha(n+\alpha-1) \xi_{2} \xi_{1}^{2}-\alpha \xi_{2}^{2}-\gamma(n+2 \alpha) \xi_{2} \xi_{1} \xi_{0}-\gamma(\gamma-1) \xi_{0}^{2} \xi_{2} \\
& +\lambda_{1}\left[3 \xi_{2} \xi_{1}^{2}+(n+\alpha-2) \xi_{1}^{4}+\gamma \xi_{1}^{3} \xi_{0}\right] \\
& +\lambda_{2}\left[2 \xi_{2} \xi_{1} \xi_{0}+(n+\alpha-1) \xi_{1}^{3} \xi_{0}+(\gamma-1) \xi_{1}^{2} \xi_{0}^{2}\right] \\
& +\lambda_{3}\left[\xi_{2} \xi_{0}^{2}+(n+\alpha) \xi_{1}^{2} \xi_{0}^{2}+(\gamma-2) \xi_{1} \xi_{0}^{3}\right] \\
& +\lambda_{4}\left[(n+\alpha+1) \xi_{1} \xi_{0}^{3}+(\gamma-3) \xi_{0}^{4}\right] \\
& \geqslant v \xi_{0}^{4}
\end{aligned}
$$


is satisfied for some constant $v>0$. W.l.o.g. we may assume that $\xi_{0}=1$ (otherwise divide the inequality by $\xi_{0}^{4}$ and substitute $\tilde{\xi}_{1}:=\xi_{0}^{-1} \xi_{1}$ and $\tilde{\xi}_{2}:=\xi_{0}^{-2} \xi_{2}$ ). Thus we only have to check whether

$$
\begin{aligned}
& -\alpha(n+\alpha-1) \xi_{2} \xi_{1}^{2}-\alpha \xi_{2}^{2}-\gamma(n+2 \alpha) \xi_{2} \xi_{1}-\gamma(\gamma-1) \xi_{2} \\
& +\lambda_{1}\left[3 \xi_{2} \xi_{1}^{2}+(n+\alpha-2) \xi_{1}^{4}+\gamma \xi_{1}^{3}\right] \\
& +\lambda_{2}\left[2 \xi_{2} \xi_{1}+(n+\alpha-1) \xi_{1}^{3}+(\gamma-1) \xi_{1}^{2}\right] \\
& +\lambda_{3}\left[\xi_{2}+(n+\alpha) \xi_{1}^{2}+(\gamma-2) \xi_{1}\right] \\
& +\lambda_{4}\left[(n+\alpha+1) \xi_{1}+(\gamma-3)\right] \\
& -v \quad \geqslant 0
\end{aligned}
$$

holds for all $\xi_{1}, \xi_{2}$ if we choose $\alpha, \gamma, \lambda_{1}, \ldots, \lambda_{4}$ and $v>0$ appropriately.

Suppose now that we have found an admissible choice of $\alpha, \gamma, \lambda_{1}, \ldots, \lambda_{4}, v$. Then we have

$$
\left.\frac{1}{1+\alpha} \int u^{1+\alpha}|x-y|^{\gamma} d x\right|_{t_{1}} ^{t_{2}} \geqslant v \int_{t_{1}}^{t_{2}} \int u^{n+\alpha+1}|x-y|^{\gamma-4} d x
$$

for any $t_{2}>t_{1} \geqslant 0$ with $t_{2}<\hat{T}_{y}^{r}$ for some $r>0$. In case $\gamma+4 \frac{1+\alpha}{n}+1<0$, we infer an upper bound on $\hat{T}_{y}^{r}$ by the considerations preceding (7). In contrast, in case $\gamma+4 \frac{1+\alpha}{n}+1>0$ we obtain the following theorem:

THEOREM 2 Let $n \in(1,2)$. Let $u$ be a strong solution to the Cauchy problem for the onedimensional thin-film equation with compactly supported nonnegative initial data $u_{0}$. Suppose that the solution has been constructed by the usual approximation procedure (see e.g. [2, 3]) or that the solution is a strong energy solution (for the terminology see e.g. [15]).

Let $x_{0} \in \partial \operatorname{supp} u_{0}$ and $\operatorname{supp} u_{0} \cap\left(x_{0}-\delta, x_{0}\right)=\emptyset$ for some $\delta>0$. Assume moreover that $u_{0}(x) \geqslant S \cdot\left(x-x_{0}\right)_{+}^{\theta}$ holds for any $x \in B_{\delta}\left(x_{0}\right)$ for some $\theta>0$ and some $S>0$.

If we can find $\alpha \in(-1,0), \gamma<0, v>0$ with $\gamma+4 \frac{1+\alpha}{n}+1>0$ such that (12) holds, then in case $\theta \leqslant \frac{-\gamma-1}{1+\alpha}$ the free boundary at $x_{0}$ starts moving forward instantaneously, i.e. we have $\inf \left\{t>0: \operatorname{supp} u(., t) \cap\left(x_{0}-\delta, x_{0}\right) \neq \emptyset\right\}=0$.

Proof. Let $y \in\left(x_{0}-\delta, x_{0}\right)$. We may apply Hölder's inequality to the right-hand side of (12) and the finite speed of propagation property to obtain

$$
\begin{aligned}
\left.\int u^{1+\alpha}|x-y|^{\gamma} d x\right|_{t_{1}} ^{t_{2}} & \\
& \geqslant v \int_{t_{1}}^{t_{2}}\left(\int_{\operatorname{supp} u(., t)}|x-y|^{\gamma+4 \frac{1+\alpha}{n}} d x\right)^{-\frac{n}{1+\alpha}}\left(\int u^{1+\alpha}|x-y|^{\gamma} d x\right)^{\frac{1+\alpha+n}{1+\alpha}} d t \\
& \geqslant c\left(n, \alpha, \gamma, u_{0}\right) \int_{t_{1}}^{t_{2}}\left(\int u^{1+\alpha}|x-y|^{\gamma} d x\right)^{\frac{1+\alpha+n}{1+\alpha}} d t
\end{aligned}
$$

for $0 \leqslant t_{1}<t_{2}<\min \left(1, T_{y}^{\left|x_{0}-y\right|}\right)$. This implies by the differential inequality argument

$$
\min \left(1, T_{y}^{\left|x_{0}-y\right|}\right) \leqslant C\left(n, \alpha, \gamma, u_{0}\right)\left(\int u^{1+\alpha}|x-y|^{\gamma} d x\right)^{-\frac{n}{1+\alpha}} .
$$


Set $y=x_{0}-\epsilon$. Letting $\epsilon \searrow 0$, we infer the following estimate for the waiting time at $x_{0}$ (note that for $x>x_{0}+\epsilon$ we have $\left.\left|x-x_{0}+\epsilon\right| \leqslant 2\left|x-x_{0}\right|\right)$ :

$$
\min \left(1, T^{*}\right) \leqslant C\left(n, \alpha, \gamma, u_{0}\right) \liminf _{\epsilon \rightarrow 0}\left(\int_{\left(x_{0}+\epsilon, x_{0}+\delta\right)} S^{1+\alpha}\left|x-x_{0}\right|^{\theta(1+\alpha)} \cdot\left|x-x_{0}\right|^{\gamma} d x\right)^{-\frac{n}{1+\alpha}}
$$

In case $\theta \leqslant \frac{-\gamma-1}{1+\alpha}$, this implies our theorem.

\subsection{Numerical results}

The quantifier elimination problem from the previous subsection cannot be solved exactly by current algorithms. However, we may try to solve the system numerically (which leads to a loss of optimality, but not to a loss of rigorousness). We first observe that the left-hand side of the inequality is a second-order polynomial with respect to $\xi_{2}$. Since we have $\alpha<0$ we see that the parabola tends to infinity as $\left|\xi_{2}\right| \rightarrow \infty$ for any fixed $\xi_{1}$. Freezing $\xi_{1}$, we see that the parabola is nonnegative if and only if the discriminant is nonpositive, i.e. if and only if

$$
\begin{aligned}
& {\left[-\alpha(n+\alpha-1) \xi_{1}^{2}-\gamma(n+2 \alpha) \xi_{1}-\gamma(\gamma-1)+3 \lambda_{1} \xi_{1}^{2}+2 \lambda_{2} \xi_{1}+\lambda_{3}\right]^{2}} \\
& +4 \alpha\left[\lambda_{1}(n+\alpha-2) \xi_{1}^{4}+\lambda_{1} \gamma \xi_{1}^{3}+\lambda_{2}(n+\alpha-1) \xi_{1}^{3}+\lambda_{2}(\gamma-1) \xi_{1}^{2}\right. \\
& \left.+\lambda_{3}(n+\alpha) \xi_{1}^{2}+\lambda_{3}(\gamma-2) \xi_{1}+\lambda_{4}(n+\alpha+1) \xi_{1}+\lambda_{4}(\gamma-3)-v\right]
\end{aligned}
$$

In this inequality the left-hand side is the fourth-order polynomial

$$
\begin{aligned}
& {\left[4 \alpha \lambda_{1}(n+\alpha-2)+\left(-\alpha(n+\alpha-1)+3 \lambda_{1}\right)^{2}\right] \xi_{1}^{4}} \\
& +\left[2\left(-\alpha(n+\alpha-1)+3 \lambda_{1}\right)\left(-\gamma(n+2 \alpha)+2 \lambda_{2}\right)+4 \alpha \lambda_{1} \gamma+4 \alpha \lambda_{2}(n+\alpha-1)\right] \xi_{1}^{3} \\
& +\left[2\left(-\alpha(n+\alpha-1)+3 \lambda_{1}\right)\left(-\gamma(\gamma-1)+\lambda_{3}\right)+\left(-\gamma(n+2 \alpha)+2 \lambda_{2}\right)^{2}\right. \\
& \left.+4 \alpha \lambda_{2}(\gamma-1)+4 \alpha \lambda_{3}(n+\alpha)\right] \xi_{1}^{2} \\
& +\left[2\left(-\gamma(n+2 \alpha)+2 \lambda_{2}\right)\left(-\gamma(\gamma-1)+\lambda_{3}\right)+4 \alpha \lambda_{3}(\gamma-2)+4 \alpha \lambda_{4}(n+\alpha+1)\right] \xi_{1} \\
& +\left[\left(-\gamma(\gamma-1)+\lambda_{3}\right)^{2}+4 \alpha \lambda_{4}(\gamma-3)-4 \alpha \nu\right] .
\end{aligned}
$$

Note that we now just need to find $\alpha, \gamma, \lambda_{1}, \ldots, \lambda_{4}$ such that this polynomial is strictly negative in case $v=0$; then we can always find some $v>0$ such that the resulting polynomial is still nonpositive.

Checking whether a fourth-order polynomial is nonnegative can be done, e.g., using the following test (as shown in the paper by Ulrich and Watson [21]):

THEOREM 3 (Ulrich and Watson [21]) Given a quartic polynomial $f(z)=a z^{4}+b z^{3}+c z^{2}+d z+e$ with $a>0$ and $e>0$, set

$$
\alpha:=b a^{-\frac{3}{4}} e^{-\frac{1}{4}},
$$




$$
\begin{aligned}
\beta & :=c a^{-\frac{1}{2}} e^{-\frac{1}{2}}, \\
\gamma & :=d a^{-\frac{1}{4}} e^{-\frac{3}{4}}, \\
\Delta & :=4\left(\beta^{2}-3 \alpha \gamma+12\right)^{3}-\left(72 \beta+9 \alpha \beta \gamma-2 \beta^{3}-27 \alpha^{2}-27 \gamma^{2}\right)^{2}, \\
\Lambda_{1} & :=(\alpha-\gamma)^{2}-16(\alpha+\beta+\gamma+2), \\
\Lambda_{2} & :=(\alpha-\gamma)^{2}-\frac{4(\beta+2)}{\sqrt{\beta-2}}(\alpha+\gamma+4 \sqrt{\beta-2}) .
\end{aligned}
$$

Then $f(z) \geqslant 0$ for all $z \geqslant 0$ if and only if one of the following conditions holds:

- We have $\beta<-2$ and $\Delta \leqslant 0$ and $\alpha+\gamma>0$.

- It holds that $-2 \leqslant \beta \leqslant 6$ and $\left((\Delta \leqslant 0\right.$ and $\alpha+\gamma>0)$ or $\left(\Delta \geqslant 0\right.$ and $\left.\left.\Lambda_{1} \leqslant 0\right)\right)$.

- We have $\beta>6$ and $\left((\Delta \leqslant 0\right.$ and $\alpha+\gamma>0)$ or $(\alpha>0$ and $\gamma>0)$ or $\left(\Delta \geqslant 0\right.$ and $\left.\Lambda_{2} \leqslant 0\right)$ ).

We now check using the previous theorem whether the polynomial (13) is strictly negative; to do so, we sample a part of the domain of definition of the mapping $\left(\alpha, \gamma, \lambda_{1}, \ldots, \lambda_{4}\right) \mapsto$ Polynomial(...). The sampled intervals and the corresponding step size are shown in Table 1 below; the maximal growth exponent $\theta$ for which we can prove instantaneous forward motion of the interface (see Theorem 2) is stated, along with the parameters for which it has been found.

For example, in the case $n=1.9$ we checked for all $\alpha \in\{-0.32,-0.31, \ldots,-0.20\}$, all $\gamma \in\{-2.6,-2.55, \ldots,-2.00\}$, all $\lambda_{1} \in\{-0.3,-0.277, \ldots, 0.229\}, \ldots$, whether the polynomial (13) is strictly negative. For each such tuple $\left(\alpha, \gamma, \lambda_{1}, \ldots, \lambda_{4}\right)$ for which negativity holds, Theorem 2 implies instantaneous forward motion of the free boundary if the initial data grow steeper than $\left(x-x_{0}\right)_{+}^{\theta}, \theta=\frac{-\gamma-1}{1+\alpha}$. For our $n=1.9$, the tuple with the largest corresponding $\theta$ among our sampled tuples which has a negative corresponding polynomial is $\left(\alpha, \gamma, \lambda_{1}, \ldots, \lambda_{4}\right)=$ $(-0.27,-2.25,-0.07, \ldots,-12.6)$; for this choice, we have $\theta=1.7123$.

Let us compare our new results to the following values for the maximal growth exponent $\theta$ which have been obtained by direct calculations in [15] (or at least follow from the conditions on $n$, $\alpha$, and $\gamma$ in this paper using a computer algebra program):

\begin{tabular}{ccccc}
\hline$n$ & $\alpha$ & $\gamma$ & Old Exponent $\theta$ & Improved Exponent $\theta$ \\
\hline 1.90 & -0.451 & -1.900 & 1.640 & 1.7123 \\
1.80 & -0.400 & -1.792 & 1.320 & 1.4588 \\
1.70 & -0.351 & -1.685 & 1.055 & 1.2210 \\
1.60 & -0.301 & -1.576 & 0.825 & 1.0024 \\
1.50 & -0.252 & -1.469 & 0.627 & 0.7997 \\
1.40 & -0.203 & -1.362 & 0.454 & 0.5823 \\
1.30 & -0.153 & -1.260 & 0.306 & 0.4575 \\
1.20 & -0.104 & -1.168 & 0.187 & 0.2900 \\
1.10 & -0.0517 & -1.078 & 0.055 & 0.1812 \\
\hline
\end{tabular}

The existence of the stationary solution $u(x, t):=x_{+}^{2}$ places a restriction on the possible values of $\alpha \in(-1,0]$ and $\gamma<0$ for which monotonicity formulas of the form

$$
\frac{d}{d t} \int u^{1+\alpha}|x-y|^{\gamma} d x \geqslant c \int u^{1+\alpha+n}|x-y|^{\gamma-4} d x
$$

may be deduced using our approach: Take a cutoff $\psi$ with $\psi \equiv 1$ for $x<K$ and $\psi \equiv 0$ for $x>2 K$. If we can prove a monotonicity formula as above using our approach, we can also prove a 
TABLe 1.

\begin{tabular}{|c|c|c|c|c|c|c|c|c|c|}
\hline$n$ & \multicolumn{5}{|c|}{$\alpha, \gamma, \lambda_{1}, \lambda_{2}, \lambda_{3}, \lambda_{4}$} & Growth Exponent $\theta$ & \multicolumn{3}{|c|}{ Parameters } \\
\hline 1.9 & $\begin{array}{l}\alpha \\
\gamma \\
\lambda_{1} \\
\lambda_{2} \\
\lambda_{3} \\
\lambda_{4}\end{array}$ & $\begin{array}{l}= \\
= \\
= \\
= \\
= \\
=\end{array}$ & $\begin{array}{c}-0.32 \\
-2.6 \\
-0.3 \\
-2.5 \\
8.8 \\
-14\end{array}$ & $\begin{array}{l}0.01 \\
0.05 \\
00.023 \\
\vdots 0.035 \\
0.027 \\
0.10\end{array}$ & $\begin{array}{c}-0.20 \\
-2.00 \\
0.25 \\
-0.9 \\
11.3 \\
-11\end{array}$ & 1.7123 & $\begin{array}{l}\alpha \\
\nu \\
\lambda_{1} \\
\lambda_{2} \\
\lambda_{3} \\
\lambda_{4}\end{array}$ & $\begin{array}{l}= \\
= \\
= \\
= \\
= \\
=\end{array}$ & $\begin{array}{c}-0.27 \\
-2.25 \\
-0.07 \\
-2.185 \\
9.043 \\
-12.6\end{array}$ \\
\hline 1.8 & $\begin{array}{l}\alpha \\
\gamma \\
\lambda_{1} \\
\lambda_{2} \\
\lambda_{3} \\
\lambda_{4}\end{array}$ & $\begin{array}{l}= \\
= \\
= \\
= \\
= \\
=\end{array}$ & $\begin{array}{c}-0.35 \\
-2.4 \\
-0.3 \\
-2.5 \\
8.5 \\
-14\end{array}$ & $\begin{array}{l}: 0.013 \\
: 0.05: \\
: 0.023 \\
00.035 \\
: 0.027 \\
: 0.10\end{array}$ & $\begin{array}{c}-0.20 \\
-1.80 \\
0.25 \\
-0.9 \\
11.0 \\
-11\end{array}$ & 1.4588 & $\begin{array}{l}\alpha \\
\gamma \\
\lambda_{1} \\
\lambda_{2} \\
\lambda_{3} \\
\lambda_{4}\end{array}$ & $\begin{array}{l}= \\
= \\
= \\
= \\
= \\
=\end{array}$ & $\begin{array}{c}-0.246 \\
-2.1 \\
-0.07 \\
-2.15 \\
8.959 \\
-12.2\end{array}$ \\
\hline 1.7 & $\begin{array}{l}\alpha \\
\nu \\
\lambda_{1} \\
\lambda_{2} \\
\lambda_{3} \\
\lambda_{4}\end{array}$ & $\begin{array}{l}= \\
= \\
= \\
= \\
= \\
=\end{array}$ & $\begin{array}{c}-0.30 \\
-2.25 \\
-0.3 \\
-2.5 \\
8.5 \\
-14\end{array}$ & $\begin{array}{c}: 0.017 \\
: 0.05: \\
\vdots 0.025 \\
00.035 \\
: 0.033 \\
: 0.12:\end{array}$ & $\begin{array}{c}-0.17 \\
-1.70 \\
0.25 \\
-0.9 \\
11.0 \\
-11\end{array}$ & 1.2210 & $\begin{array}{l}\alpha \\
\nu \\
\lambda_{1} \\
\lambda_{2} \\
\lambda_{3} \\
\lambda_{4}\end{array}$ & $\begin{array}{l}= \\
= \\
= \\
= \\
= \\
=\end{array}$ & $\begin{array}{c}-0.222 \\
-1.95 \\
-0.05 \\
-2.185 \\
8.962 \\
-11.84\end{array}$ \\
\hline 1.6 & $\begin{array}{l}\alpha \\
\nu \\
\lambda_{1} \\
\lambda_{2} \\
\lambda_{3} \\
\lambda_{4}\end{array}$ & $\begin{array}{l}= \\
= \\
= \\
= \\
= \\
=\end{array}$ & $\begin{array}{c}-0.27 \\
-2.02 \\
-0.1 \\
-2.5 \\
8.0 \\
-14\end{array}$ & $\begin{array}{c}: 0.013 \\
: 0.0525 \\
: 0.01 \\
00.035 \\
00.033 \\
: 0.12\end{array}$ & $\begin{array}{c}-0.15 \\
-1.60 \\
0.05 \\
-0.9 \\
10.5 \\
-10\end{array}$ & 1.0024 & $\begin{array}{l}\alpha \\
\nu \\
\lambda_{1} \\
\lambda_{2} \\
\lambda_{3} \\
\lambda_{4}\end{array}$ & $\begin{array}{l}= \\
= \\
= \\
= \\
= \\
=\end{array}$ & $\begin{array}{c}-0.192 \\
-1.81 \\
-0.03 \\
-2.115 \\
8.462 \\
-11.00\end{array}$ \\
\hline 1.5 & $\begin{array}{c}\alpha \\
\nu \\
\lambda_{1} \\
\lambda_{2} \\
\lambda_{3} \\
\lambda_{4} \\
\alpha \\
\gamma \\
\lambda_{1} \\
\lambda_{2} \\
\lambda_{3} \\
\lambda_{4}\end{array}$ & $\begin{array}{l}= \\
= \\
= \\
= \\
= \\
= \\
= \\
= \\
= \\
= \\
= \\
=\end{array}$ & $\begin{array}{c}-0.24 \\
-1.9 \\
-0.07 \\
-2.5 \\
7.0 \\
-14 \\
-0.22 \\
-1.9 \\
-0.07 \\
-2.5 \\
7.0 \\
-10.5\end{array}$ & $\begin{array}{l}: 0.014 \\
00.045 \\
00.008 \\
00.037 \\
0.035 \\
: 0.12 \\
00.014 \\
00.045 \\
00.005 \\
00.037 \\
0.035 \\
0.12\end{array}$ & $\begin{array}{c}-0.12 \\
-1.50 \\
0.04 \\
-1.3 \\
9.5 \\
-9.7 \\
-0.08 \\
-1.4 \\
0.04 \\
-1.3 \\
9.5 \\
-9.0\end{array}$ & 0.5823 & $\begin{array}{c}\alpha \\
\nu \\
\lambda_{1} \\
\lambda_{2} \\
\lambda_{3} \\
\lambda_{4} \\
\alpha \\
\nu \\
\lambda_{1} \\
\lambda_{2} \\
\lambda_{3} \\
\lambda_{4}\end{array}$ & $\begin{array}{l}= \\
= \\
= \\
= \\
= \\
= \\
= \\
= \\
= \\
= \\
= \\
=\end{array}$ & $\begin{array}{c}-0.156 \\
-1.675 \\
-0.022 \\
-1.908 \\
7.63 \\
-10.04 \\
-0.15 \\
-1.495 \\
-0.01 \\
-1.871 \\
7.35 \\
-9.3\end{array}$ \\
\hline 1.3 & $\begin{array}{l}\alpha \\
\gamma \\
\lambda_{1} \\
\lambda_{2} \\
\lambda_{3} \\
\lambda_{4}\end{array}$ & $\begin{array}{l}= \\
= \\
= \\
= \\
= \\
=\end{array}$ & $\begin{array}{c}-0.18 \\
-1.6 \\
-0.05 \\
-2.2 \\
5.0 \\
-10.1\end{array}$ & \begin{tabular}{c}
$: 0.014$ \\
00.045 \\
\hdashline 0.003 \\
00.037 \\
\hdashline 0.035 \\
0.12
\end{tabular} & $\begin{array}{c}-0.05 \\
-1.2 \\
0.02 \\
-1.2 \\
9.0 \\
-6.8\end{array}$ & 0.4575 & $\begin{array}{l}\alpha \\
\gamma \\
\lambda_{1} \\
\lambda_{2} \\
\lambda_{3} \\
\lambda_{4}\end{array}$ & $\begin{array}{l}= \\
= \\
= \\
= \\
= \\
=\end{array}$ & $\begin{array}{c}-0.082 \\
-1.42 \\
-0.008 \\
-1.423 \\
5.665 \\
-7.58\end{array}$ \\
\hline 1.2 & $\begin{array}{l}\alpha \\
\nu \\
\lambda_{1} \\
\lambda_{2} \\
\lambda_{3} \\
\lambda_{4}\end{array}$ & $\begin{array}{l}= \\
= \\
= \\
= \\
= \\
= \\
=\end{array}$ & $\begin{array}{c}-0.15 \\
-1.5 \\
-0.05 \\
-2.1 \\
4.1 \\
-8.5\end{array}$ & \begin{tabular}{c}
$: 0.014$ \\
0.045 \\
00.003 \\
\hdashline 0.037 \\
\hdashline 0.035 \\
$: 0.12$
\end{tabular} & $\begin{array}{c}-0.02 \\
-1.1 \\
0.02 \\
-0.9 \\
7.0 \\
-5.8\end{array}$ & 0.2900 & $\begin{array}{l}\alpha \\
\nu \\
\lambda_{1} \\
\lambda_{2} \\
\lambda_{3} \\
\lambda_{4}\end{array}$ & $\begin{array}{l}= \\
= \\
= \\
= \\
= \\
=\end{array}$ & $\begin{array}{c}-0.052 \\
-1.275 \\
-0.005 \\
-1.138 \\
4.52 \\
-6.22\end{array}$ \\
\hline 1.1 & $\begin{array}{l}\alpha \\
\gamma \\
\lambda_{1} \\
\lambda_{2} \\
\lambda_{3} \\
\lambda_{4} \\
\end{array}$ & $\begin{array}{l}= \\
= \\
= \\
= \\
= \\
=\end{array}$ & $\begin{array}{c}-0.04 \\
-1.3 \\
-0.015 \\
-1.5 \\
2.5 \\
-6.5\end{array}$ & $\begin{array}{l}: 0.003 \\
: 0.02 \\
: 0.001 \\
0.037 \\
0.035 \\
: 0.12:\end{array}$ & $\begin{array}{c}-0.00 \\
-1.0 \\
0.005 \\
-0.3 \\
5.1 \\
-3.0\end{array}$ & 0.1812 & $\begin{array}{l}\alpha \\
\nu \\
\lambda_{1} \\
\lambda_{2} \\
\lambda_{3} \\
\lambda_{4} \\
\end{array}$ & $\begin{array}{l}= \\
= \\
= \\
= \\
= \\
=\end{array}$ & $\begin{array}{c}-0.007 \\
-1.18 \\
-0.001 \\
-0.723 \\
2.885 \\
-4.22 \\
\end{array}$ \\
\hline
\end{tabular}

formula of the form

$$
\frac{d}{d t} \int u^{1+\alpha}|x-y|^{\gamma} \psi d x \geqslant c \int_{(0, K)} u^{1+\alpha+n}|x-y|^{\gamma-4} d x-C(K) \int_{(K, 2 K)} u^{1+\alpha+n}|x-y|^{\gamma} d x
$$

which yields in the case of the stationary solution for any $T>0$ and any $\epsilon>0$ (by setting $y:=-\epsilon$ )

$$
c T \int_{(0, K)} x_{+}^{2(1+\alpha+n)}|x+\epsilon|^{\gamma-4} d x-C(K) T \int_{(K, 2 K)} x_{+}^{2(1+\alpha+n)}|x+\epsilon|^{\gamma} d x \leqslant 0 .
$$

The second term in this estimate is either bounded uniformly with respect to $\epsilon>0$ or it is at least negligible in comparison with the first term in case of $\epsilon>0$ small enough. Letting $\epsilon \rightarrow 0$, we see 
that we must have $2(1+\alpha+n)+\gamma-4>-1$ as otherwise the first term in this estimate would blow up. Rearranging, we deduce that the exponent $\theta$ sufficient for the nonexistence of a waiting time is bounded from above by $\theta=\frac{-\gamma-1}{1+\alpha} \leqslant \frac{2 n+2 \alpha-2}{1+\alpha}=2-\frac{4-2 n}{1+\alpha}$; by $\alpha \in(-1,0]$ this yields $\theta \leqslant 2 n-2$. We see that our exponents in the previous table are fairly close to this theoretical limit of our method.

In the paper [15], upper bounds on waiting times have only been proven for $n \in\left(2, \frac{32}{11}\right)$, while one would expect it to be possible for any $n \in(2,3)$. Numerical evidence obtained by our method suggest that this is indeed the case; the following table lists admissible values for $\alpha, \gamma, \lambda_{1}, \ldots, \lambda_{4}$ which we have found for $n \in\left[\frac{32}{11}, 3\right)$ using our computer-based analysis:

\begin{tabular}{ccccccc}
\hline$n$ & $\alpha$ & $\gamma$ & $\lambda_{1}$ & $\lambda_{2}$ & $\lambda_{3}$ & $\lambda_{4}$ \\
\hline 2.93 & -0.96 & -2 & -0.331 & -1.448 & 7.06 & -11 \\
2.94 & -0.97 & -2 & -0.331 & -1.448 & 6.78 & -11 \\
2.95 & -0.97 & -2 & -0.331 & -1.296 & 6.55 & -10.3 \\
2.96 & -0.97 & -2 & -0.331 & -1.054 & 5.535 & -9.4 \\
2.97 & -0.98 & -2 & -0.331 & -1.092 & 5.785 & -9.5 \\
2.98 & -0.99 & -2 & -0.331 & -1.092 & 5.575 & -9.5 \\
2.99 & -0.995 & -2 & -0.331 & -0.94 & 4.91 & -8.9 \\
2.995 & -0.998 & -2 & -0.331 & -0.864 & -4.65 & -8.6 \\
2.999 & -0.9995 & -2 & -0.331 & -0.716 & 4.035 & -8.0 \\
\hline
\end{tabular}

The idea for establishing the monotonicity formulas which is suggested by these results - namely to use the first and the second formula for integrating by parts to deal with terms of the form $\int u^{n+\alpha-2} u_{x x}\left|u_{x}\right|^{2}|x-y|^{\gamma} d x-$ is used in [12] to extend the results of [13, 15] to the range $n \in\left[\frac{32}{11}, 3\right)$.

\section{Application to a sixth-order analogue of the Derrida-Lebowitz-Speer-Spohn equation}

In this section, we apply a variant of our method to the one-dimensional sixth-order equation

$$
\frac{d}{d t} u=\left[u\left(\frac{1}{u}\left(u(\log u)_{x x}\right)_{x x}+\frac{1}{2}\left((\log u)_{x x}\right)^{2}\right)_{x}\right]_{x}
$$

as considered by Jüngel and Milisic [19]. Weak solutions on $\Omega:=[0,1)$ with periodic boundary conditions (as defined in [19]) are required to satisfy $u \in W_{l o c}^{1,1}\left(I ;\left[H_{p e r}^{3}(\Omega)\right]^{\prime}\right), \sqrt{u} \in$ $L_{l o c}^{2}\left(I ; H_{p e r}^{3}(\Omega)\right)$ as well as $u^{\frac{1}{6}} \in L_{l o c}^{6}\left(I ; W_{p e r}^{1,6}(\Omega)\right)$, and to satisfy the equation

$$
\begin{aligned}
\int_{0}^{T}\left\langle u_{t}, \psi\right\rangle d t & \\
=\int_{0}^{T} \int_{\Omega}-2 \sqrt{u}(\sqrt{u})_{x x x} \psi_{x x x} & +\frac{32}{3} u^{\frac{1}{4}}\left(\left(u^{\frac{1}{4}}\right)_{x}\right)^{3} \psi_{x x x}-12 \sqrt{u}\left|\left(u^{\frac{1}{4}}\right)_{x}\right|^{2} \psi_{x x x x} \\
& +8\left|(\sqrt{u})_{x x}\right|^{2} \psi_{x x}+\frac{128}{3}\left|\left(u^{\frac{1}{4}}\right)_{x}\right|^{4} \psi_{x x} d x d t
\end{aligned}
$$

for any $\psi \in L_{l o c}^{\infty}\left(I ; H_{p e r}^{3}(\Omega)\right)$ and any $T>0$. We shall prove the following theorem: 
THEOREM 4 Let $u$ be a weak solution to the sixth-order equation (14) on $\Omega:=[0,1)$ with nonvanishing (nonnegative) initial data $u_{0}$ and periodic boundary conditions. Let $x_{0} \in \Omega$. Define $T:=\inf \left\{t \geqslant 0: \operatorname{dist}\left(x_{0}, \bigcup_{\tau \in[0, t]} \operatorname{supp} u(., \tau)\right)=0\right\}$, where dist denotes the periodicity-adjusted distance. Then we have $T \leqslant \frac{1}{247000}$.

Proof. The proof proceeds by combining the ideas from [14] with the algorithmic construction of entropies. W.l.o.g. we may assume that $x_{0}=0$. Note that (by periodicity) $u$ vanishes identically for $t<T$ on some neighbourhood of both 0 and 1 .

Let $\epsilon>0$. Set $\psi:=|x+\epsilon|^{\gamma+2}$ (where $\gamma<-2$ ). For any $\delta>0$, this function coincides on $(0+\delta, 1-\delta)$ with a smooth compactly supported function $\psi_{\delta} \in C_{c p t}^{\infty}\left(\left(\frac{\delta}{2}, 1-\frac{\delta}{2}\right)\right)$ (to see this, just multiply $\psi$ with a cutoff which is equal to one in $(\delta, 1-\delta)$ and zero outside of $\left.\left(\frac{\delta}{2}, 1-\frac{\delta}{2}\right)\right)$. Thus we may plug in $\psi_{\delta}$ as a test function in (15) and replace immediately $\psi_{\delta}$ by $\psi$ in the resulting equation since for $\delta>0$ small enough $\psi_{\delta} \neq \psi$ holds only on the set where the solution $u$ vanishes. Integrating by parts in the first term on the right-hand side of the resulting equation, we obtain

$$
\begin{aligned}
& \left.\int_{\Omega} u(., t)|x+\epsilon|^{\gamma+2} d x\right|_{t_{1}} ^{t_{2}} \\
& =(\gamma+2)(\gamma+1) \int_{t_{1}}^{t_{2}} \int_{\Omega} 2(\sqrt{u})_{x}(\sqrt{u})_{x x}\left(|x+\epsilon|^{\gamma}\right)_{x}+2 \sqrt{u}(\sqrt{u})_{x x}\left(|x+\epsilon|^{\gamma}\right)_{x x} \\
& +\frac{32}{3} u^{\frac{1}{4}}\left(\left(u^{\frac{1}{4}}\right)_{x}\right)^{3}\left(|x+\epsilon|^{\gamma}\right)_{x}-12 \sqrt{u}\left|\left(u^{\frac{1}{4}}\right)_{x}\right|^{2}\left(|x+\epsilon|^{\gamma}\right)_{x x} \\
& +8\left|(\sqrt{u})_{x x}\right|^{2}|x+\epsilon|^{\gamma}+\frac{128}{3}\left|\left(u^{\frac{1}{4}}\right)_{x}\right|^{4}|x+\epsilon|^{\gamma} d x d t
\end{aligned}
$$

for any $t_{1}, t_{2} \in[0, T)$. We thus get (note that we have $\nabla \sqrt{u}=0$ a.e. on $\{u=0\}$ since $\sqrt{u} \in H^{1}$ for a.e. $t>0$; for this implication, see, e.g., Chapter 5.10 in [11])

$$
\begin{aligned}
\frac{1}{(\gamma+2)(\gamma+1)} & \left.\int_{\Omega} u(., t)|x+\epsilon|^{\gamma+2} d x\right|_{t_{1}} ^{t_{2}} \\
= & \int_{t_{1}}^{t_{2}} \int_{\Omega} 2 \gamma(\sqrt{u})_{x}(\sqrt{u})_{x x}|x+\epsilon|^{\gamma-1}-2 \gamma(\gamma-1)\left|{ }_{u}\right|^{2}|x+\epsilon|^{\gamma-2} d x d t \\
& +\int_{t_{1}}^{t_{2}} \int_{\Omega} \gamma(\gamma-1)(\gamma-2)(\gamma-3) \sqrt{u}^{2}|x+\epsilon|^{\gamma-4} d x d t \\
& +\int_{t_{1}}^{t_{2}} \int_{\Omega} \frac{4}{3} \gamma \frac{1}{\sqrt{u}}\left((\sqrt{u})_{x}\right)^{3}|x+\epsilon|^{\gamma-1} d x d t \\
& +\int_{t_{1}}^{t_{2}} \int_{\Omega}-3 \gamma(\gamma-1)\left|(\sqrt{u})_{x}\right|^{2}|x+\epsilon|^{\gamma-2} d x d t \\
& +\int_{t_{1}}^{t_{2}} \int_{\Omega} 8\left|(\sqrt{u})_{x x}\right|^{2}|x+\epsilon|^{\gamma}+\frac{8}{3} \frac{1}{\sqrt{u}^{2}}\left|(\sqrt{u})_{x}\right|^{4}|x+\epsilon|^{\gamma} d x d t
\end{aligned}
$$

Integration by parts yields the identities

$\int_{\Omega} \frac{1}{\sqrt{u}^{2}}\left|\sqrt{u}_{x}\right|^{4}|x+\epsilon|^{\gamma} d x=3 \int_{\Omega} \frac{1}{\sqrt{u}}\left|\sqrt{u}_{x}\right|^{2} \sqrt{u}_{x x}|x+\epsilon|^{\gamma} d x+\gamma \int_{\Omega} \frac{1}{\sqrt{u}}\left(\sqrt{u}_{x}\right)^{3}|x+\epsilon|^{\gamma-1} d x$ 
(this formula holds for strictly positive $\sqrt{u} \in H^{2}(\Omega)$ which are constant in some neighbourhood of 0 and 1; for nonnegative $\sqrt{u}$ which vanish in some neighbourhood of 0 and 1 , it holds by approximation. For the approximation procedure see e.g. [14]) and

$$
2 \int_{\Omega}(\sqrt{u})_{x}(\sqrt{u})_{x x}|x+\epsilon|^{\gamma-1} d x=-(\gamma-1) \int_{\Omega}\left|\sqrt{u}_{x}\right|^{2}|x+\epsilon|^{\gamma-2} d x
$$

as well as

$$
\int_{\Omega}\left|\sqrt{u}_{x}\right|^{2}|x+\epsilon|^{\gamma-2} d x=-\int_{\Omega} \sqrt{u} \sqrt{u}_{x x}|x+\epsilon|^{\gamma-2} d x-(\gamma-2) \int_{\Omega} \sqrt{u} \sqrt{u}_{x}|x+\epsilon|^{\gamma-3} d x
$$

and

$$
2 \int_{\Omega} \sqrt{u} \sqrt{u}_{x}|x+\epsilon|^{\gamma-3} d x=-(\gamma-3) \int_{\Omega} \sqrt{u}^{2}|x+\epsilon|^{\gamma-4} d x
$$

Introducing the abbreviations $\xi_{1}$ for $\frac{1}{\sqrt{u}} \sqrt{u}, \xi_{2}$ for $\frac{1}{\sqrt{u}} \sqrt{u_{x x}}$, and $\xi_{0}$ for $|x+\epsilon|^{-1}$, these formulas can be rewritten as

$$
\int_{\Omega}\left(\xi_{1}^{4}-3 \xi_{2} \xi_{1}^{2}-\gamma \xi_{1}^{3} \xi_{0}\right) u|x+\epsilon|^{\gamma} d x=0
$$

and

$$
\int_{\Omega}\left(2 \xi_{1} \xi_{2} \xi_{0}+(\gamma-1) \xi_{1}^{2} \xi_{0}^{2}\right) u|x+\epsilon|^{\gamma} d x=0
$$

as well as

$$
\int_{\Omega}\left(\xi_{2} \xi_{0}^{2}+\xi_{1}^{2} \xi_{0}^{2}+(\gamma-2) \xi_{1} \xi_{0}^{3}\right) u|x+\epsilon|^{\gamma} d x=0
$$

and

$$
\int_{\Omega}\left(2 \xi_{1} \xi_{0}^{3}+(\gamma-3) \xi_{0}^{4}\right) u|x+\epsilon|^{\gamma} d x=0 .
$$

We now try to find $\lambda_{1}, \ldots, \lambda_{4} \in \mathbb{R}$ and $\gamma<-2, K>0$ such that for any $\xi_{0}>0$ and any $\xi_{1}, \xi_{2} \in \mathbb{R}$ the estimate

$$
\begin{aligned}
2 \gamma \xi_{2} \xi_{1} \xi_{0}-2 & \gamma(\gamma-1) \xi_{1}^{2} \xi_{0}^{2}+\gamma(\gamma-1)(\gamma-2)(\gamma-3) \xi_{0}^{4} \\
& +\frac{4}{3} \gamma \xi_{1}^{3} \xi_{0}-3 \gamma(\gamma-1) \xi_{1}^{2} \xi_{0}^{2}+8 \xi_{2}^{2}+\frac{8}{3} \xi_{1}^{4}-K \xi_{0}^{4} \\
& +\lambda_{1}\left(\xi_{1}^{4}-3 \xi_{2} \xi_{1}^{2}-\gamma \xi_{1}^{3} \xi_{0}\right)+\lambda_{2}\left(2 \xi_{1} \xi_{2} \xi_{0}+(\gamma-1) \xi_{1}^{2} \xi_{0}^{2}\right) \\
& +\lambda_{3}\left(\xi_{2} \xi_{0}^{2}+\xi_{1}^{2} \xi_{0}^{2}+(\gamma-2) \xi_{1} \xi_{0}^{3}\right)+\lambda_{4}\left(2 \xi_{1} \xi_{0}^{3}+(\gamma-3) \xi_{0}^{4}\right)
\end{aligned}
$$

holds. If it is possible for some $K>0$ and $\gamma<-2$ to find such $\lambda_{1}, \ldots, \lambda_{4}$, then by (16) the estimate

$$
\left.\frac{1}{(\gamma+2)(\gamma+1)} \int_{\Omega} u(., t)|x+\epsilon|^{\gamma+2} d x\right|_{t_{1}} ^{t_{2}} \geqslant K \int_{t_{1}}^{t_{2}} \int_{\Omega} u(., t)|x+\epsilon|^{\gamma-4} d x d t
$$


is valid for all $0 \leqslant t_{1}<t_{2}<T$. This implies

$$
\left.\frac{1}{(\gamma+2)(\gamma+1)} \int_{\Omega} u(., t)|x+\epsilon|^{\gamma+2} d x\right|_{t_{1}} ^{t_{2}} \geqslant \frac{K}{(1+\epsilon)^{6}} \int_{t_{1}}^{t_{2}} \int_{\Omega} u(., t)|x+\epsilon|^{\gamma+2} d x d t
$$

which in turn by Gronwalls Lemma yields the bound

$$
\int_{\Omega} u(., T)|x+\epsilon|^{\gamma+2} d x \geqslant e^{(\gamma+1)(\gamma+2)(1+\epsilon)^{-6} K T} \int_{\Omega} u_{0}|x+\epsilon|^{\gamma+2} d x .
$$

Using conservation of mass and the fact that $\gamma<-2$, we deduce

$$
\epsilon^{\gamma+2} \int_{\Omega} u_{0} d x \geqslant e^{(\gamma+1)(\gamma+2)(1+\epsilon)^{-6} K T}(1+\epsilon)^{\gamma+2} \int_{\Omega} u_{0} d x
$$

This finally implies the estimate $\frac{(\gamma+1)(\gamma+2) K T}{(1+\epsilon)^{6}}+(\gamma+2) \log \frac{1+\epsilon}{\epsilon} \leqslant 0$, i.e. $T \leqslant \frac{1}{-K(\gamma+1)}(1+$ $\epsilon)^{6} \log \frac{1+\epsilon}{\epsilon}$. Setting $\epsilon:=0.0571109$, we get $T \leqslant \frac{4.07245}{-K(\gamma+1)}$. If we can choose $K=14204.3$ and $\gamma=-72$, then the theorem is established.

We now prove that we may indeed choose $K=14204.3$ and $\gamma=-72$. By homogeneity, we see that (17) is satisfied for all $\xi_{1}, \xi_{2}, \xi_{0}$ iff it is satisfied for $\xi_{0}=1$ and all $\xi_{1}, \xi_{2}$. Condition (17) is therefore equivalent to

$$
\begin{aligned}
2 \gamma \xi_{2} \xi_{1}-2 \gamma & (\gamma-1) \xi_{1}^{2}+\gamma(\gamma-1)(\gamma-2)(\gamma-3) \\
& +\frac{4}{3} \gamma \xi_{1}^{3}-3 \gamma(\gamma-1) \xi_{1}^{2}+8 \xi_{2}^{2}+\frac{8}{3} \xi_{1}^{4}-K \\
& +\lambda_{1}\left(\xi_{1}^{4}-3 \xi_{2} \xi_{1}^{2}-\gamma \xi_{1}^{3}\right)+\lambda_{2}\left(2 \xi_{1} \xi_{2}+(\gamma-1) \xi_{1}^{2}\right) \\
& +\lambda_{3}\left(\xi_{2}+\xi_{1}^{2}+(\gamma-2) \xi_{1}\right)+\lambda_{4}\left(2 \xi_{1}+(\gamma-3)\right)
\end{aligned}
$$

$$
\geqslant 0 \text {. }
$$

The expression on the left-hand side is a quadratic polynomial in $\xi_{2}$ with the leading-order coefficient being nonnegative; thus the expression is nonnegative for all $\xi_{1}$ and $\xi_{2}$ if and only if the discriminant is nonpositive, i.e., iff

$$
\begin{array}{r}
32 \cdot\left(-2 \gamma(\gamma-1) \xi_{1}^{2}+\gamma(\gamma-1)(\gamma-2)(\gamma-3)+\frac{4}{3} \gamma \xi_{1}^{3}-3 \gamma(\gamma-1) \xi_{1}^{2}+\frac{8}{3} \xi_{1}^{4}-K\right. \\
\left.+\lambda_{1} \xi_{1}^{4}-\lambda_{1} \gamma \xi_{1}^{3}+\lambda_{2}(\gamma-1) \xi_{1}^{2}+\lambda_{3} \xi_{1}^{2}+\lambda_{3}(\gamma-2) \xi_{1}+2 \lambda_{4} \xi_{1}+\lambda_{4}(\gamma-3)\right) \\
-\left(2 \gamma \xi_{1}-3 \lambda_{1} \xi_{1}^{2}+2 \lambda_{2} \xi_{1}+\lambda_{3}\right)^{2} \\
\geqslant 0 .
\end{array}
$$

Rearranging, we see that have to find $\lambda_{1}, \ldots, \lambda_{4} \in \mathbb{R}$ such that the fourth-order polynomial

$$
\begin{aligned}
& \left(\frac{256}{3}+32 \lambda_{1}-9 \lambda_{1}^{2}\right) \xi_{1}^{4} \\
& +\left(32 \cdot \frac{4}{3} \gamma-32 \lambda_{1} \gamma+6 \lambda_{1}\left(2 \gamma+2 \lambda_{2}\right)\right) \xi_{1}^{3}
\end{aligned}
$$




$$
\begin{aligned}
& +\left(32 \lambda_{2}(\gamma-1)-64 \gamma(\gamma-1)+32 \lambda_{3}-\left(2 \gamma+2 \lambda_{2}\right)^{2}+6 \lambda_{1} \lambda_{3}-3 \cdot 32 \gamma(\gamma-1)\right) \xi_{1}^{2} \\
& +\left(32 \lambda_{3}(\gamma-2)+64 \lambda_{4}-2 \lambda_{3}\left(2 \gamma+2 \lambda_{2}\right)\right) \xi_{1} \\
& +\left(32 \lambda_{4}(\gamma-3)+32 \gamma(\gamma-1)(\gamma-2)(\gamma-3)-\lambda_{3}^{2}-32 K\right)
\end{aligned}
$$

is nonnegative. Checking whether this polynomial has any real zero can be done e.g. with the test from [21] (i.e. Theorem 3 above). Invoking our numerical method and sampling the space of possible values for $\lambda_{1}, \ldots, \lambda_{4}$, we in particular find the solution $\gamma=-88, \lambda_{1}=3.74+\frac{4}{3}$, $\lambda_{2}=(-2.41+1) \cdot 88, \lambda_{3}=2.504 \cdot 88^{2}, \lambda_{4}=0.83 \cdot 88^{3}, K=2620$; this is the solution with the largest $|\gamma|$ which we have been able to find. Moreover, we obtain the solution $\gamma=-72$, $\lambda_{1}=3.804+\frac{4}{3}, \lambda_{2}=(-2.28+1) \cdot 72, \lambda_{3}=2.424 \cdot 72^{2}, \lambda_{4}=0.856 \cdot 72^{3}, K=14204.3$.

Note that the theorem is not entirely satisfactory; one might hope to prove infinite speed of propagation. However, our numerical results suggest that it might be impossible to prove our monotonicity formula for $\gamma<-90$. This might be overcome by considering entropies $\int u^{1+\alpha} d x$ for $\alpha \in(-1,0)$; however, for $\alpha \neq 0$ terms involving the square of the third derivative of $\sqrt{u}$ appear on the right-hand side, therefore significantly enhancing the complexity of the quantifier elimination problem to be solved.

\section{Discussion}

We have presented an algorithm for the derivation of upper bounds on waiting times for nonnegativity-preserving higher-order parabolic equations. In the case of the thin-film equation, the algorithm has been seen to lead to improved results as compared to the previous paper [15]. Even for second-order doubly nonlinear equations, our approach has lead to some improvement of the known bounds. We also have obtained a lower bound on support propagation for a sixth-order quantum drift-diffusion equation.

However, in the case of the thin-film equation with $n \in(1,2)$ our sufficient conditions for the nonexistence of a waiting time phenomenon may be suboptimal. One may hope to prove instantaneous forward motion of the free boundary for initial data growing like $x_{+}^{\beta}, \beta \in(0,2)$, at the initial free boundary; see [4] for a formal analysis of the initial behaviour of the free boundary. However, the rigorous derivation of such an improved result currently seems out of reach.

Acknowledgement. This research was supported by the Lithuanian-Swiss cooperation program under the project agreement No. CH-SMM-01/0.

\section{REFERENCES}

1. Alt, H. W., \& Luckhaus, S., Quasilinear Elliptic-Parabolic Differential Equations, Math. Z. 183 (1983), 311-341. Zb10497.35049 MR0706391

2. Beretta, E., Bertsch, M., \& Passo, R. D., Nonnegative Solutions of a Fourth-Order Nonlinear Degenerate Parabolic Equation, Arch. Ration. Mech. Anal. 129 (1995), 175-200. Zb10827.35065 MR1328475

3. Bertsch, M., Passo, R. D., Garcke, H., \& Grün, G., The thin viscous flow equation in higher space dimensions, Adv. Differential Equations 3 (1998), 417-440. Zbl0954. 35035 MR1751951 
4. Blowey, J. F., King, J. R., \& LAngdon, S., Small- and waiting-time behaviour of the thin-film equation, SIAM J. Appl. Math. 67 (2007), 1776-1807. Zb11178. 35388 MR2350007

5. Bukal, M., JÜNGel, A., \& MAtthes, D., A multidimensional nonlinear sixth-order quantum diffusion equation, Ann. Inst. H. Poincaré Anal. Non Linéaire 30 (2013), 337-365. Zbl1288. 35283 MR3035980

6. Bukal, M., Jüngel, A., \& Matthes, D., Entropies for radially symmetric higher-order nonlinear diffusion equations, Commun. Math. Sci. 9 (2011), 353-382. Zbl1221. 35077 MR2815676

7. Chipot, M., \& Sideris, T. An Upper Bound For The Waiting Time For Nonlinear Degenerate Parabolic Equations. Trans. Amer. Math. Soc. 288 (1985), 423-427. Zb10535. 35049 MR0773069

8. Dal Passo, R., Giacomelli, L., \& GRÜN, G., A waiting time phenomenon for thin film equations, Ann. Sc. Norm. Super. Pisa Cl. Sci. (4) 30 (2001), 437-463. Zbl1024. 35051 MR1895718

9. Diaz, J. I., \& Veron, L., Local vanishing properties of solutions of elliptic and parabolic quasilinear equations, Trans. Amer. Math. Soc. 290 (1985), 787-814. Zb10579. 35003 MR0792828

10. DJIE, K. C., An upper bound for the waiting time for doubly nonlinear parabolic equations, Interfaces Free Bound. 9 (2007), 95-105. Zbl1117. 35317 MR2317300

11. Evans, L. C., Partial Differential Equations, Graduate Studies in Mathematics. American Mathematical Society, 2010. Zbl1194.35001 MR2597943

12. FISCHER, J., Behaviour of free boundaries in thin-film flow: the regime of strong slippage and the regime of very weak slippage, Preprint (2013).

13. FISCHER, J., Optimal lower bounds on asymptotic support propagation rates for the thin-film equation, $J$. Differential Equations 255 (2013), 3127-3149. MR3093359

14. FISCHER, J., Infinite speed of support propagation for the Derrida-Lebowitz-Speer-Spohn equation and quantum drift-diffusion models, NoDEA Nonlinear Differential Equations Appl. 21 (2014), 27-50. Zb106358111 MR3158799

15. Fischer, J., Upper bounds on waiting times for the thin-film equation: the case of weak slippage, Arch. Ration. Mech. Anal. 2113 (2014), 771-818. Zbl1293. 35241 MR3158807

16. Giacomelli, L., \& GRÜN, G., Lower bounds on waiting times for degenerate parabolic equations and systems, Interfaces Free Bound. 8 (2006), 111-129. Zbl1100 . 35058 MR2231254

17. JÜNGEl, A., \& MATthes, D. An algorithmic construction of entropies in higher-order nonlinear pdes, Nonlinearity 19 (2006), 633-659. Zb11091. 35031 MR2209292

18. Jüngel, A., Matthes, D., \& Toscani, G., Convex sobolev inequalities derived from entropy dissipation, Arch. Ration. Mech. Anal. 199 (2011), 563-596. Zbl1229. 35302 MR2763034

19. JÜNGEL, A., \& MILISIC, J.-P., A sixth-order nonlinear parabolic equation for quantum systems, SIAM J. Math. Anal. 41 (2009), 1472-1490. Zbl1197. 35151 MR2556571

20. Shishrov, A. E., \& Shchelkov, A. Dynamics of the supports of energy solutions of mixed problems for quasi-linear parabolic equations of arbitrary order. Izvestiya: Mathematics 62 (1998), 601-626. Zb10918.35075 MR1642176

21. Ulrich, G., \& Watson, L. T., Positivity conditions for quartic polynomials, SIAM J. Sci. Comput. 15 (1994), 528-544. Zb10805.65012 MR1273150 